\title{
Lower bound equilibrium element and submodel for shear joints in precast concrete structures
}

Herfelt, Morten Andersen; Poulsen, Peter Noe; Hoang, Linh Cao; Jensen, Jesper F.

Published in:

Engineering Structures

Link to article, DOI:

10.1016/j.engstruct.2016.12.054

Publication date:

2017

Document Version

Peer reviewed version

Link back to DTU Orbit

Citation (APA):

Herfelt, M. A., Poulsen, P. N., Hoang, L. C., \& Jensen, J. F. (2017). Lower bound equilibrium element and submodel for shear joints in precast concrete structures. Engineering Structures, 135, 1-9.

https://doi.org/10.1016/j.engstruct.2016.12.054

\section{General rights}

Copyright and moral rights for the publications made accessible in the public portal are retained by the authors and/or other copyright owners and it is a condition of accessing publications that users recognise and abide by the legal requirements associated with these rights.

- Users may download and print one copy of any publication from the public portal for the purpose of private study or research.

- You may not further distribute the material or use it for any profit-making activity or commercial gain

- You may freely distribute the URL identifying the publication in the public portal

If you believe that this document breaches copyright please contact us providing details, and we will remove access to the work immediately and investigate your claim 


\title{
Lower bound equilibrium element and submodel for shear joints in precast concrete structures
}

\author{
Morten A. Herfelt ${ }^{\mathrm{a}}$, Peter N. Poulsen ${ }^{\mathrm{b}}$, Linh C. Hoang ${ }^{\mathrm{b}}$, Jesper F. Jensen ${ }^{\mathrm{a}}$ \\ ${ }^{a}$ ALECTIA A/S, Teknikerbyen 34, 2830 Virum, Denmark \\ ${ }^{b}$ Department of Civil Engineering, Technical University of Denmark, Brovej, Building \\ 118, 2800 Kgs. Lyngby, Denmark
}

\begin{abstract}
This paper is concerned with the shear capacity of keyed joints reinforced with overlapping U-bar loops in the transverse direction. The layout of the loop reinforcement affects the capacity and failure mode, and currently it is not accounted for by standards or previous theoretical work. A multiscale approach to the issue is proposed: An equilibrium element for finite element limit analysis representing keyed joints is coupled with a suitable submodel, which handles the complex stress states within the joint. The submodel is based on several modified stringer models, which makes it possible to account for local mechanisms in the core of the joint. The element and submodel are validated by comparison to a detailed model based on finite element limit analysis and experimental data. The joint element and submodel lead to a small optimisation problem compared to the detailed model and the computational time is reduced by several magnitudes.
\end{abstract}

Keywords: in-situ cast joints, limit analysis, precast concrete, finite element, rigid plasticity, yield criterion, multiscale

\section{Introduction}

The lateral stability of modern precast concrete buildings is often ensured by shear walls, i.e. precast wall panels connected by in-situ cast joints. Horizontal forces, e.g. from wind load or seismic action, are transferred as in-plane forces and the shear capacity of the panels and joints are of the

Email address: mahe@alectia.com (Morten A. Herfelt) 
utmost importance. In practice, the shear capacity of such walls is usually assessed by analytical lower bound models, e.g. strut-and-tie models or stress field methods $[1,2]$. The stress fields are also frequently determined by use of linear elastic finite element analysis. Naturally, this practice often leads to suboptimal structures compared to what can be obtained if the stress fields instead are determined from a non-linear elastic-plastic analysis. Use of numerical elastic-plastic analysis to determine stress fields has e.g. been demonstrated in References [3, 4].

The joints between the precast panels are of particular interest as they are often a critical part of the structure. In-situ cast joints consist of a concrete core and two interfaces, where the core typically is reinforced in two directions, and the interfaces typically are keyed. The shear capacity of the joints and interfaces is in practice assessed by simple empirical formulas [5] which often gives a conservative estimate of the capacity. Several authors have investigated the behaviour of in-situ cast joints. The investigations cover both experimental testing [see e.g. 6, 7, 8, 9] and simplified mechanical models based on the theory of rigid-plasticity, namely upper bound solutions based on yield line theory $[10,11]$ and lower bound solutions based on strut-and-tie models $[1,11,12]$. The experiments showed that the geometry of the joint and the reinforcement layout affect the shear capacity as well as the collapse mode, but the analytical methods have only been able to capture the observed behaviour to a certain extent. Local failure mechanisms caused by the reinforcement layout, however, have not been investigated using analytical methods. Investigations using numerical tools, e.g. finite element method or similar, have focused on single key joints often used in precast concrete segmental bridges $[13,14]$. These investigations have primarily been carried out by use of non-linear finite element analysis. This approach is computationally heavy, especially when considering the fact that the ultimate load carrying capacity is the result of main interest.

Herfelt et al. [15] presented a detailed model for keyed joints based on finite element limit analysis. The model was based on a lower bound formulation and the analysis yielded a statically admissible stress field. Moreover, the solution to the dual problem, i.e. the corresponding kinematic problem, was interpreted as the failure mode. The detailed model used triangular plane stress elements [16] representing the concrete, bar elements [16] representing the reinforcement, and an interface elements representing the concrete-to-concrete interfaces. It was shown that the model could represent the complex stress states within the joint and captured the local failure 


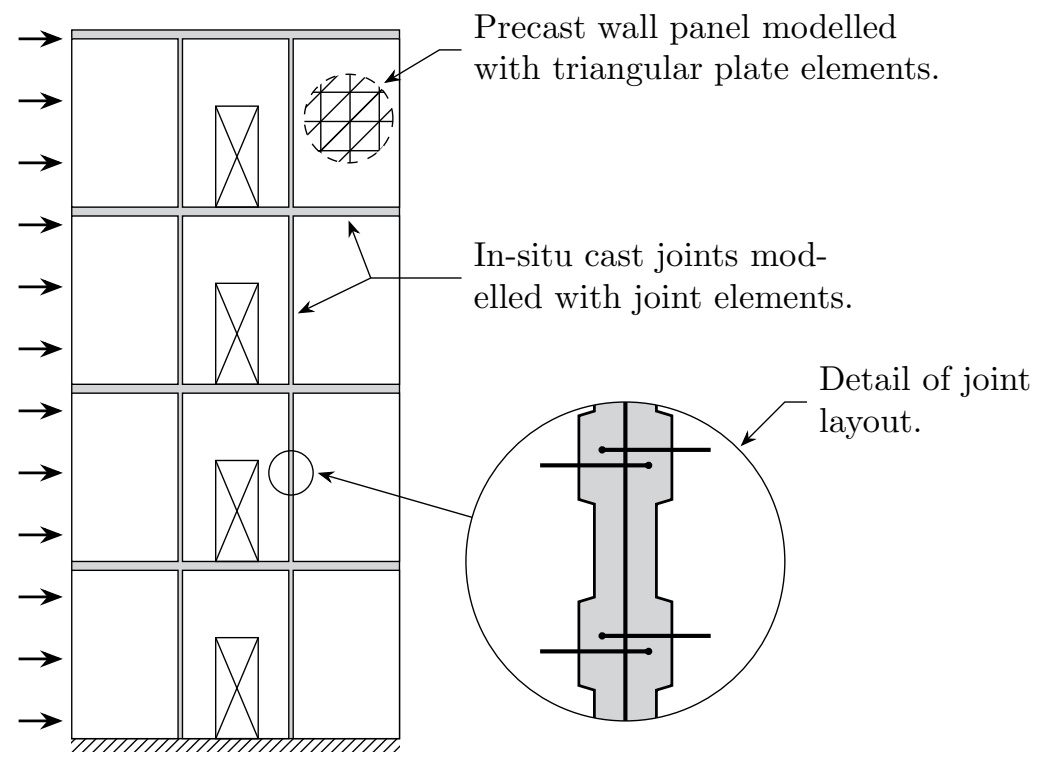

Figure 1: Four storey wall subjected to horizontal forces: The wall consists of 12 precast panels connected by joints. An example of a mesh (discretisation) for numerical analysis is seen for the top right panel.

mechanisms to a satisfactory degree; however, for practical design it is not feasible to use that level of detail. Fig. 1 shows a four storey wall comprising several precast panels connected by in-situ cast joints. As indicated in the figure, plane stress elements may be used to model the precast panels, while a special joint element is needed for the joints.

This paper presents a lower bound equilibrium element representing the insitu cast joints. The element is designed for interaction with the triangular plane stress element [16] and interface elements [15]. The scope is to be able to model entire wall systems, e.g. the four storey wall seen in Fig. 1. The joint element requires a suitable yield criterion which can capture the critical mechanisms identified by the detailed model [15], and for this purpose, a semi-analytical submodel yield criterion based on the stringer method is developed. The joint element and submodel fit the format of second-order cone programming, and the developed model is compared to the detailed model [15] as well as experimental data [6, 7]. The proposed multiscale model captures the behaviour of the detailed model as well as the specimens. 


\section{Problem formulation}

Finite element limit analysis can be considered as a special case of the general finite element method: It is based on the extremum principles for rigid-plastic materials [see e.g. 1, 17, 18] and deploys a mesh discretisation known from the finite element method. Anderheggen and Knöpfel [19] presented a general formulation as well as equilibrium elements for solids and plates. Since the 1970s several authors have contributed to the method [see e.g. 16, 20, 21]. Finite element limit analysis is a direct method, where the ultimate load is determined in a single step, which is a significant advantage over non-linear finite element methods for practical applications. Moreover, when modelling concrete structures, there is no need to consider any tensile strength to avoid problems related to numerical stability. From the lower bound formulation, the stress field is determined. Associated with the lower bound problem is a so-called dual problem, and the solution to this dual problem can be interpreted as the displacement field and plastic strain. Since we are dealing with a rigid plastic material model, no information on the magnitude of the strains and displacements are determined; only the directions. When the method is applied to structural concrete, it is necessary to operate with effective strength parameters (via the so-called effectiveness factors) to account for the limited ductility of concrete as well as the reduction of the compressive strength as a result of cracking and tension strains transverse to compressive stress fields. In practice, the effective strength parameters have to be found by calibration of calculations with results of tests on structural components.

Numerical lower bound limit analysis is formulated as an optimisation problem where the scope is to maximise a load factor $\lambda$. The analysis determines a statically admissible stress, i.e. a stress field which satisfies equilibrium and does not violate the yield criteria in any points. The general problem can be stated as [16, 22]:

$$
\begin{array}{ll}
\text { maximise } & \lambda \\
\text { subject to } & \mathbf{H} \boldsymbol{\beta}=\boldsymbol{R} \lambda+\boldsymbol{R}_{0} \\
& f\left(\boldsymbol{\beta}_{i}\right) \leq 0, \quad i=1,2, \ldots, m
\end{array}
$$

The load acting on the model consists of a constant part $\boldsymbol{R}_{0}$ and a scalable part $\boldsymbol{R} \lambda$. The linear equality constraints ensure equilibrium while the functions $f\left(\boldsymbol{\beta}_{i}\right) \leq 0$ ensure that the stress filed does not violate the yield criteria. $\mathbf{H}$ is the global equilibrium matrix, and $\boldsymbol{\beta}$ is the stress vector. $m$ is the 
number of check points for the yield function, $f$, which is generally convex, but non-linear; thus, (1) represents a convex optimisation problem.

In this work, the optimisation problem (1) will be a second-order cone program (SOCP). Second-order cone programming as well as semidefinite programming have been used in the field of finite element limit analysis for more than a decade, [see e.g. References 23, 24, 25]. Expanding the yield functions $f,(1)$ can be restated as:

$$
\begin{array}{ll}
\text { maximise } & \lambda \\
\text { subject to } & \mathbf{H} \boldsymbol{\beta}=\boldsymbol{R} \lambda+\boldsymbol{R}_{0} \\
& \mathbf{C}_{\beta} \boldsymbol{\beta}+\mathbf{C}_{\alpha} \boldsymbol{\alpha}+\mathbf{C}_{\gamma} \boldsymbol{\gamma}=\boldsymbol{C}_{\mathbf{0}} \\
& \mathbf{E}_{\beta} \boldsymbol{\beta}+\mathbf{E}_{\alpha} \boldsymbol{\alpha}+\mathbf{E}_{\gamma} \boldsymbol{\gamma} \leq \boldsymbol{E}_{\mathbf{0}} \\
& \boldsymbol{\gamma}_{i} \in \mathcal{Q}_{k_{i}}, \quad i=1,2, \ldots, m
\end{array}
$$

where $\boldsymbol{\alpha}$ and $\boldsymbol{\gamma}$ are problem variables associated with the yield functions, and the $\mathbf{C}$ and $\mathbf{E}$ matrices define the necessary linear equalities and inequalities for the chosen yield criterion. The variables $\gamma_{i}$, associated with the $i$ th check point for the stresses, are in a quadratic cone $\mathcal{Q}_{k_{i}}$ of size $k_{i}$, defined as:

$$
\mathcal{Q}_{k}=\left\{\boldsymbol{x} \mid \boldsymbol{x} \in \mathbb{R}^{k}, x_{1} \geq \sqrt{x_{2}^{2}+\cdots+x_{k}^{2}}\right\}
$$

The problem (2) can be solved efficiently using interior point algorithm, and in this work the commercial solver MOSEK [26] is used. The reader is referred to References $[27,28,29,30]$ for a detailed description of SOCP and interior point solvers.

On the element level, the equilibrium equations and yield functions can be stated as:

$$
\begin{array}{ll}
\mathbf{h}_{e l} \boldsymbol{\beta}_{e l}=\boldsymbol{q}_{e l} & \\
\mathbf{C}_{\beta}^{e l, i} \boldsymbol{\beta}_{e l}+\mathbf{C}_{\alpha}^{e l, i} \boldsymbol{\alpha}_{i}+\mathbf{C}_{\gamma}^{e l, i} \boldsymbol{\gamma}_{i}=\boldsymbol{C}_{\mathbf{0}}^{e l, i}, & i=1,2, \ldots, m_{e l} \\
\mathbf{E}_{\beta}^{e l, i} \boldsymbol{\beta}_{e l}+\mathbf{E}_{\alpha}^{e l, i} \boldsymbol{\alpha}_{i}+\mathbf{E}_{\gamma}^{e l, i} \boldsymbol{\gamma}_{i} \leq \boldsymbol{E}_{\mathbf{0}}^{e l, i}, & i=1,2, \ldots, m_{e l} \\
\boldsymbol{\gamma}_{i} \in \mathcal{Q}_{k_{i}}, & i=1,2, \ldots, m_{e l}
\end{array}
$$

$\boldsymbol{q}_{e l}$ is the contributions to the equilibrium equations on the global level, $\boldsymbol{\beta}_{e l}$ is the stress variables of the given element, and $\mathbf{h}_{e l}$ is the element equilibrium matrix. The matrices $\mathbf{C}^{e l, i}$ and $\mathbf{E}^{e l, i}$ define the yield function for the $m_{e l}$ check points of the element. Similarly to (2), the variables denoted $\gamma_{i}$ are required to be in a quadratic cone $Q_{k_{i}}$ of size $k_{i}$. 


\section{Keyed joints and detailed numerical model}

A keyed joint reinforced with loop reinforcement (U-bars) and a locking bar is considered. Fig. 2 shows the basic geometry and a unit section (dashed rectangle) which will form the foundation of the submodel yield criterion. The thick vertical lines seen in Fig. 2 represent the loop reinforcement, while the horizontal solid line represents the locking bar. The length of keyed joints in practice usually ranges from a single storey height to the height of the entire building, while the width $b$ and thickness $t$ usually are below 200 $\mathrm{mm}$.

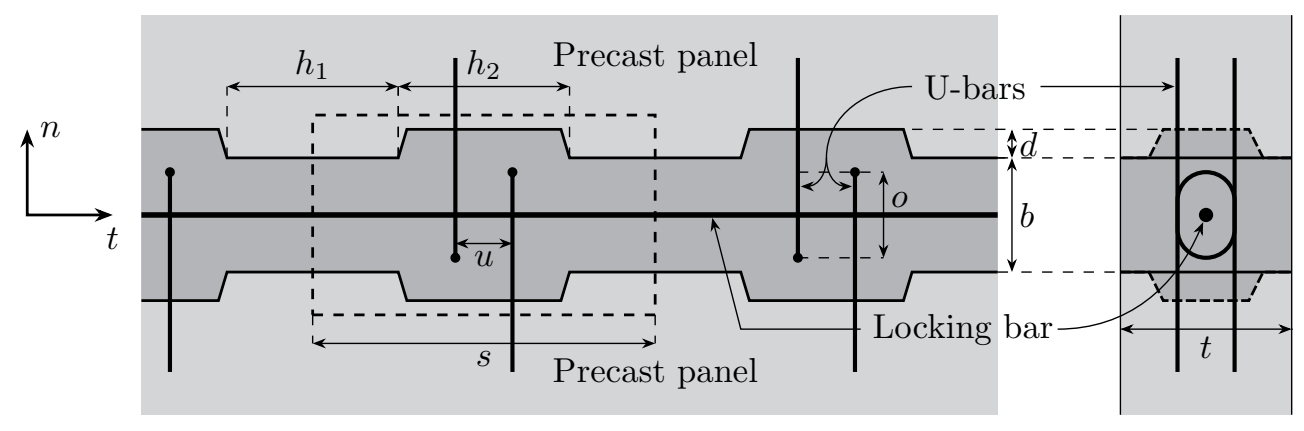

Figure 2: Elevation and cross section of a basic design of a keyed joint reinforced with U-bars.

For the detailed numerical model presented by Herfelt et al.[15], several thousand plane stress elements were necessary to capture the local mechanisms and stress fields developed in the core of the joint. The model was loaded such that the centre line of the joint would be subjected to pure shear, i.e. no bending. The concrete is modelled as a Mohr-Coulomb material, while a simple linear criterion is used for the rebars. For the interface, a Coulomb friction model is assumed. The model assumed plane stress state, thus, the confinement provided by the reinforcement loops and general triaxial stress states were disregarded.

Fig. 3(a) shows an example of the collapse mode determined by the aforementioned detailed model [15] using the solution to the dual problem, i.e. the corresponding kinematic problem. The interpreted velocities are associated with the equilibrium equations, hence, each element edge moves independently. The blue lines indicate the interface between the joint and the precast panels, while the red lines indicate the reinforcement. The critical mechanism features a diagonal yield line through the core of the joint. Fig. 


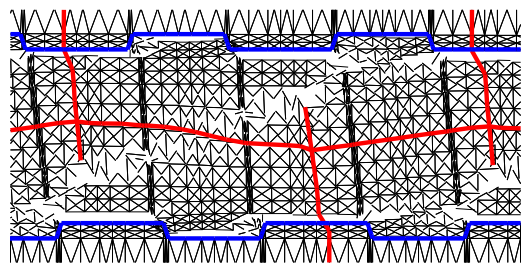

(a)

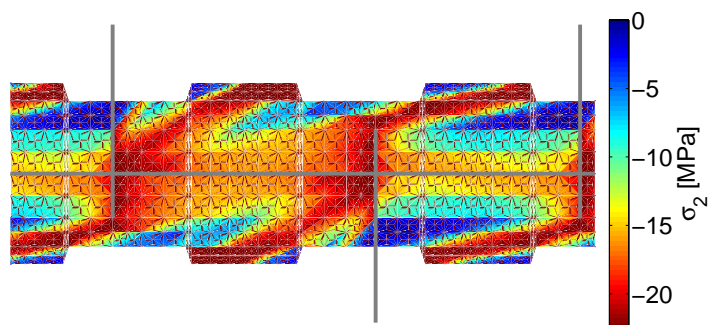

(b)

Figure 3: Detailed numerical model [15] for specimen 26 by Hansen and Olesen [6]: Collapse mode (a) and lowest principal stress (b).

3(b) shows the lowest principal stress. The reinforcement is indicated by gray lines and it is observed that large compressive stresses are present in the area between the vertical U-bars. This also shows that the reinforcement layout has a significant effect on the capacity and failure mode of the joint [15]. The detailed model [15] will be used for comparison in the following analysis.

\section{Joint equilibrium element}

An equilibrium element representing in-situ cast joints is needed for practical modelling of precast concrete structures. The joint element will dictate the distribution and transfer of stresses through the joint. The element is formulated to be compatible with the plane stress element [16], see in Fig. 4.

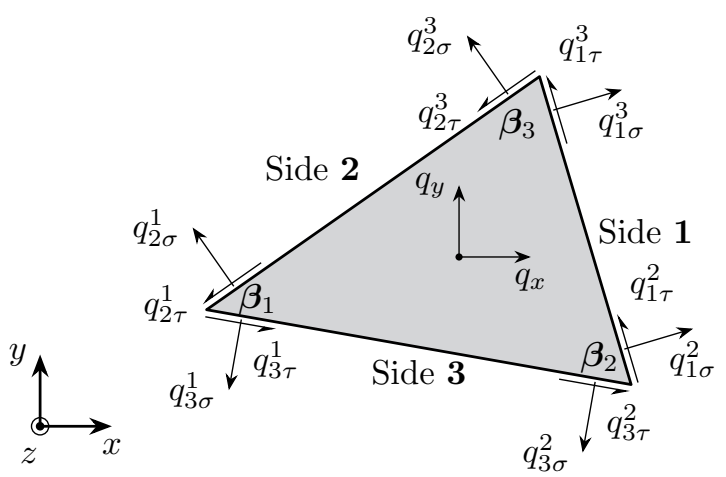

Figure 4: Generalised nodal forces and stress variables of the plane stress triangle (adapted from [16]). 
The plane stress triangle has a linear stress field defined by the three stress vectors in the corners. Equilibrium of tractions along the element boundaries means that the joint element requires a linear variation of the shear stress and transverse normal stress, which again leads to a quadratic variation of the longitudinal normal stress, see Fig. 5(b).

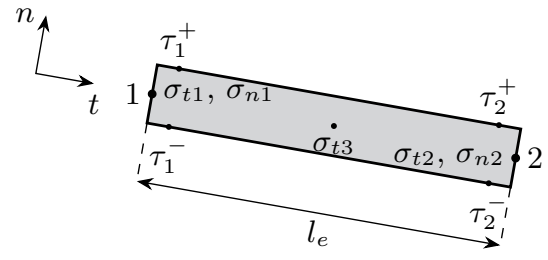

(a)

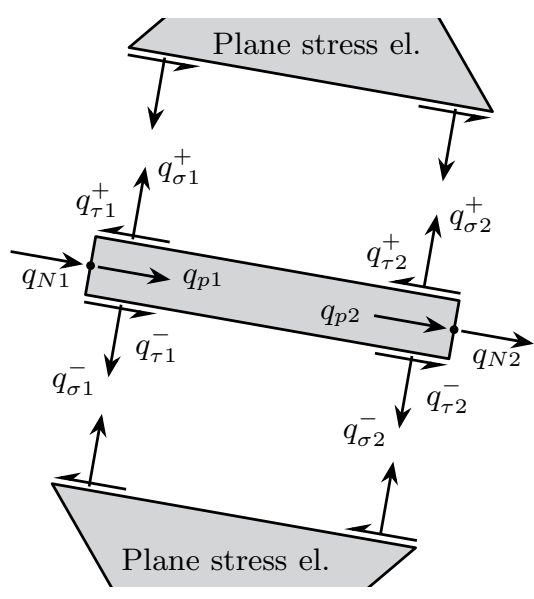

(b)

Figure 5: a) Geometry, local coordinate system, and stress variables of the joint element. b) Nodal forces of the joint element and interaction with the plane stress elements.

Fig. 5 shows the joint element. Length and orientation of the element are defined by the two end nodes seen in the figure. The element has 9 stress parameters and contributes to 12 equilibrium equation on the global level. Equilibrium for the element can be stated as:

$$
\boldsymbol{q}_{e l}=\mathbf{h}_{e l} \boldsymbol{\beta}_{e l}
$$

where $\boldsymbol{\beta}_{e l}$ is the element stress vector, $\boldsymbol{q}_{\boldsymbol{l}}$ contains the 12 nodal forces seen in Fig. 5(b), and $\mathbf{h}_{e l}$ is the local equilibrium matrix. The stress variables are given in local coordinates; thus, no transformations are necessary when 
establishing $\mathbf{h}_{e l} \cdot \boldsymbol{\beta}_{e l}, \boldsymbol{q}_{e l}$, and $\mathbf{h}_{e l}$ are explicitly given in (6):

$$
\boldsymbol{q}_{e l}=\left[\begin{array}{c}
\boldsymbol{q}^{+} \\
\boldsymbol{q}^{-} \\
\boldsymbol{q}_{N}
\end{array}\right]=\left[\begin{array}{c}
q_{\sigma 1}^{+} \\
q_{\tau 1}^{+} \\
q_{\sigma 2}^{+} \\
q_{\tau 2}^{+} \\
-q_{\sigma 1}^{-} \\
q_{\tau 1}^{-} \\
q_{\sigma 2}^{-} \\
q_{\tau 2}^{-} \\
-q_{N 1}^{-} \\
q_{N 2} \\
q_{p 1} \\
q_{p 2}
\end{array}\right]=\left[\begin{array}{ccccccccc}
t & 0 & 0 & 0 & 0 & 0 & 0 & 0 & 0 \\
0 & 0 & -t & 0 & 0 & 0 & 0 & 0 & 0 \\
0 & t & 0 & 0 & 0 & 0 & 0 & 0 & 0 \\
0 & 0 & 0 & -t & 0 & 0 & 0 & 0 & 0 \\
--t & 0 & 0 & 0 & 0 & 0 & 0 & 0 & 0 \\
0 & 0 & 0 & 0 & t & 0 & 0 & 0 & 0 \\
0 & -t & 0 & 0 & 0 & 0 & 0 & 0 & 0 \\
0 & 0 & 0 & 0 & 0 & t & 0 & 0 & 0 \\
-0 & 0 & 0 & 0 & 0 & 0 & b t & 0 & 0 \\
0 & 0 & 0 & 0 & 0 & 0 & 0 & -b t & 0 \\
0 & 0 & t & 0 & -t & 0 & 3 \frac{b t}{l_{e}} & \frac{b t}{l_{e}} & -4 \frac{b t}{l_{e}} \\
0 & 0 & 0 & t & 0 & -t & -\frac{b t}{l_{e}} & -3 \frac{b t}{l_{e}} & 4 \frac{b t}{l_{e}}
\end{array}\right]\left[\begin{array}{c}
\sigma_{n 1} \\
\sigma_{n 2} \\
\tau_{1}^{+} \\
\tau_{2}^{+} \\
\tau_{1}^{-} \\
\tau_{2}^{-} \\
\sigma_{t 1} \\
\sigma_{t 2} \\
\sigma_{t 3}
\end{array}\right]=\mathbf{h}_{e l} \boldsymbol{\beta}_{e l}
$$

The equilibrium matrix and generalised nodal force vector can be split into three parts; one for the positive side of the joint (denoted $\boldsymbol{q}^{+}$), one for the negative side of the joint (denoted $\boldsymbol{q}^{-}$), and one for the nodal forces in the longitudinal direction (denoted $\boldsymbol{q}_{N}$ ). It is assumed that the transverse normal stress ( $n$-direction) is transferred directly through the joint, while the shear stress can be used to build up an axial force in the longitudinal direction.

Each point along the joint element has a stress state defined by four stress parameters, namely $\sigma_{n}, \sigma_{t}, \tau^{+}$, and $\tau^{-}$, which passed on and checked against the appropriate model on the submodel level.

\section{Submodel yield criterion}

The stresses on the element level need to satisfy the submodel yield criterion on the joint section scale. The submodel yield criterion is in itself a small optimisation problem, and equilibrium of the submodel is ensured by stress fields that are determined by using a variation of the stringer method [see e.g. 1, 31].

The scope of the submodel yield criterion is to capture the critical mechanisms within the joint, and three modified stringer models are introduced for this purpose. The submodel is based on a unit joint section (see the dashed rectangle in Fig. 2) which is subjected to shear as well as axial forces in two directions. Fig. 6 shows the unit joint section and the three modified stringer models which compose the submodel yield criterion. Each of the three modified stringer models imposes certain constraints on the stress distribution 
in the joint and represents a basic state of stresses. These stress states are then added together to obtain the actual stress state of the combined model, which is checked against the chosen yield criteria to ensure a safe solution.

The geometry of the loop reinforcement will necessarily lead to a local, triaxial stress state in the joint; however, we have chosen to neglect this effect and assume plane stress. This assumption will - for the typical combinations of loop diameters, rebar diameters, and rebar strengths - lead to a lower shear capacity, and the joint element and submodel may underestimate the capacity in some cases.

\subsection{Equilibrium of the submodel}

In order to transfer tension or to establish confinement pressure on the joint, it is necessary to mobilise the loop reinforcement. Tension in the loop reinforcement will introduce shear in the centre row of panels, see Fig. 6(b). Based on moment equilibrium, the following relations must hold true:

$$
\tau_{21}=\frac{V}{o t}=\frac{u}{s} \frac{T}{o t}, \quad \tau_{22}=\left(\frac{u}{s}-1\right) \frac{T}{o t}
$$

Moreover, from the antisymmetric model it can be concluded that $\tau_{21}=\tau_{23}$. The stringer force $V$ seen in Fig. 6(b) will be balanced out by an adjacent joint element.

The horizontal boundaries may be subjected to shear stresses of different magnitudes, which leads to a linear varying normal force in the central stringer seen in Fig. 6(c). A rather simple stringer model is used to describe this behaviour, and the shear stresses in panels 1 and 3, Fig. 6(c), can be stated as:

$$
\tau_{1}=\tau^{+}, \quad \tau_{3}=\tau^{-}
$$

The locking bar (see Fig. 2) functions as the central stringer. Horizontal equilibrium for the stringer forces of the central stringer and two shear forces gives the following relation:

$$
F_{l t}^{+}-F_{l t}^{-}=s t\left(\tau_{1}-\tau_{3}\right)
$$

Shear panels 1 and 3, Fig. 6(c), overlap the three panels from the first stringer model, Fig. 6(b), and the resulting stresses can be determined by simply adding the stress states.

The shear panels need confinement pressure in order to carry shear stresses. The confinement pressure may originate from externally applied loads on the 
(a)

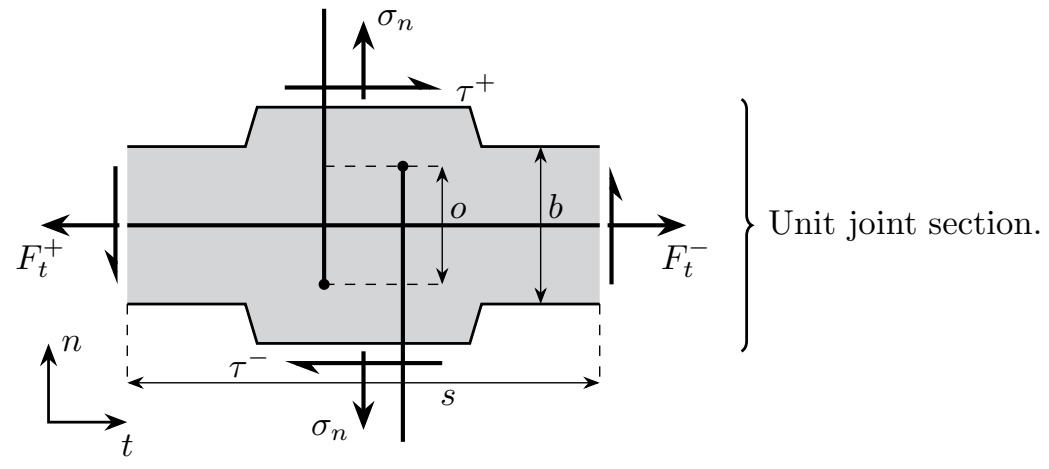

(b)

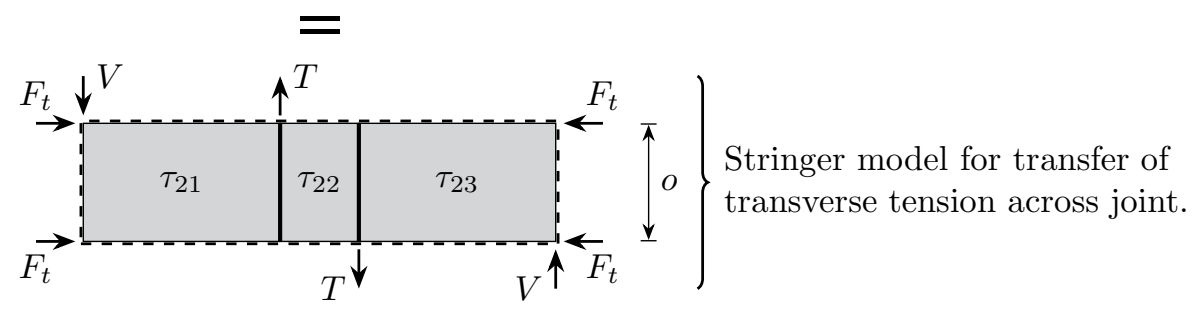

$$
\begin{aligned}
& \stackrel{(s-u) / 2}{\longleftrightarrow} \vec{u} \underset{(s-u) / 2}{\longleftrightarrow} \\
& +
\end{aligned}
$$

(c)

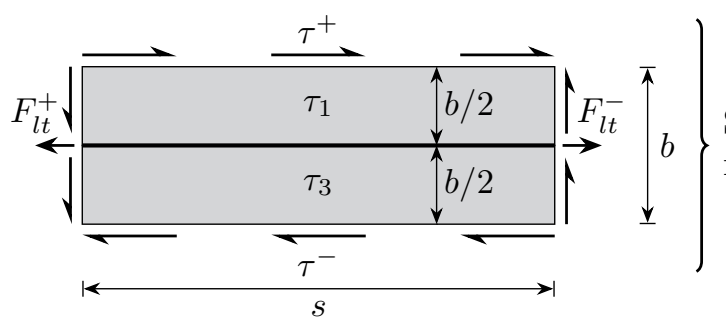

Stringer model for shear transfer across joint.

(d)

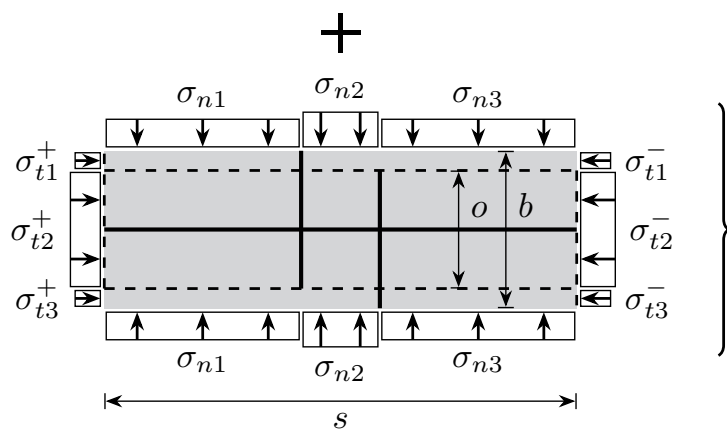

Model for transfer of compression across joint.

Figure 6: Unit joint section and the three stringer models: The behaviour of the unit joint section is divided into three main mechanisms, namely transverse tension, shear, and compression. 
unit joint section, or from stresses developed to create internal equilibrium with the tensile forces in the loop reinforcement or locking bar. The stringer model for transfer of compression, see Fig. 6(d), dictates how compressive loads are transferred through the joint. Equilibrium is required for the horizontal boundaries of the unit joint section; the transverse normal stress $\sigma_{n}$ is balanced by the stringer forces $T$ and $V$ as well as the confinement pressure:

$$
\text { st } \sigma_{n}=T-V-\frac{s-u}{2} t\left(\sigma_{n 1}+\sigma_{n 3}\right)-u t \sigma_{n 2}
$$

and similarly for the horizontal forces:

$$
\begin{aligned}
& F_{t}^{+}=F_{l t}^{+}-2 F_{t}-\frac{b-o}{2} t\left(\sigma_{t 1}^{+}+\sigma_{t 3}^{+}\right)-o t \sigma_{t 2}^{+} \\
& F_{t}^{-}=F_{l t}^{-}-2 F_{t}-\frac{b-o}{2} t\left(\sigma_{t 1}^{-}+\sigma_{t 3}^{-}\right)-o t \sigma_{t 2}^{-}
\end{aligned}
$$

The shear stress will be symmetric about the vertical center line of the unit joint section; hence, the confinement pressure in the $n$-direction will be symmetric as well, i.e. $\sigma_{n 1}=\sigma_{n 3}$. Moreover, for simplicity we assume the same for the confinement in the $t$-direction, why may lower the shear capacity in cases where $\tau^{+} \neq \tau^{-}$.

\subsection{Yield conditions for the submodel components}

The actual stress states in the panels and stringers are obtained by adding the stress states of the three stringer models, see Fig. 6(b)-(d). The actual shear stress in-between the two U-bars will e.g. be given as the sum of $\tau_{1}$ and $\tau_{22}$. The obtained stress states are required to satisfy the appropriate yield criteria as discussed in this section.

The loop reinforcement and locking bar carry tensile stringer forces, which are limited by the yield strength of the reinforcement. Moreover, we assume that the reinforcement only carries tension, thus, the yield criteria can be stated as:

$$
\begin{aligned}
& 0 \leq T \leq A_{s u} f_{Y u} \\
& 0 \leq F_{l t}^{+} \leq A_{s l} f_{Y l} \\
& 0 \leq F_{l t}^{-} \leq A_{s l} f_{Y l}
\end{aligned}
$$

where $A_{s u}$ and $A_{s l}$ are the cross sectional areas of the loop reinforcement and locking bar, respectively, and $f_{Y u}$ and $f_{Y l}$ are the yield strengths.

The horizontal stringers seen in the stringer model for transverse tension, 


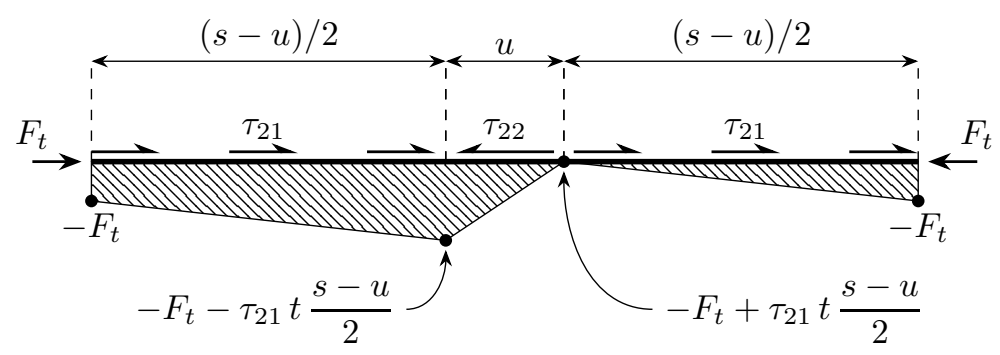

Figure 7: Compression stringer, confinement pressure $F_{t}$, and stringer force distribution.

Fig. 6(b), are so-called compression stringers without any reinforcement to carry tensile forces. The force $F_{t}$ in Fig. 7 ensures that the stringer force is non-positive. The criterion can be written as a linear inequality:

$$
-F_{t}+\tau_{21} \frac{s-u}{2} t \leq 0
$$

Finally, the shear panels need an adequate yield criterion. The stress state in each panel is described by two normal stresses and a shear stress. We use the Mohr-Coulomb yield criterion for plane stress with a tension cut-off which is given as follows in principal stresses:

$$
\begin{aligned}
\sigma_{1} & \leq f_{t} \\
k \sigma_{1}-\sigma_{2} & \leq f_{c} \\
-\sigma_{2} & \leq f_{c}
\end{aligned}
$$

where $f_{t}$ is the uniaxial tensile strength of the joint concrete, and $f_{c}$ is the effective uniaxial compressive strength. $k$ is a friction parameter defined as

$$
k=\left(\sqrt{\mu^{2}+1}+\mu\right)^{2}
$$

with $\mu=\tan \theta$, where $\theta$ is the angle of internal friction. For monolithic concrete, $\theta$ is usually taken as $37^{\circ}$, which corresponds to $k=4 . \sigma_{1}$ and $\sigma_{2}$ are the largest and smallest principal stresses, respectively, given as:

$$
\left.\begin{array}{l}
\sigma_{1} \\
\sigma_{2}
\end{array}\right\}=\frac{\sigma_{n}+\sigma_{t}}{2} \pm \sqrt{\left(\frac{\sigma_{n}-\sigma_{t}}{2}\right)^{2}+\tau_{n t}^{2}}
$$

Introducing

$$
p_{m}=-\frac{\sigma_{n}+\sigma_{t}}{2}, \sigma_{d}=\frac{\sigma_{n}-\sigma_{t}}{2}, \varphi \geq \sqrt{\sigma_{d}^{2}+\tau_{n t}^{2}},
$$


the principal stresses can now be written as:

$$
\begin{aligned}
\sigma_{1} & \leq-p_{m}+\varphi \\
-\sigma_{2} & \leq p_{m}+\varphi
\end{aligned}
$$

and the Mohr-Coulomb criterion (14) can be stated as:

$$
\begin{aligned}
-p_{m}+\varphi & \leq f_{t} \\
(1-k) p_{m}+(k+1) \varphi & \leq f_{c} \\
p_{m}+\varphi & \leq f_{c}
\end{aligned}
$$

The yield criterion fits the format of second-order cone programming since the definition of $\varphi$ (16) is a quadratic cone, see Eq. 3, while the the three constrains in (17) as well as the definitions of $p_{m}$ and $\sigma_{d}(16)$ are linear.

\section{Analysis and discussion}

\subsection{Model}

In the following, the results obtained using a single joint element with the submodel yield criterion will be compared to the results of detailed model [15]. The shear load sought to be maximised is applied as on both sides of the joint element, see $q_{\tau 1}^{+}, q_{\tau 1}^{-}, q_{\tau 2}^{+}$, and $q_{\tau 2}^{-}$in Fig. 5(b), and no normal forces are introduced via the external loading, see Fig. 8.

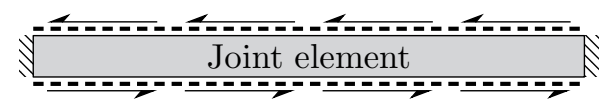

Figure 8: Model for the analysis including supports and loading. The dashed lines indicates the interface elements representing the keyed interface.

In practice, the keys near the ends of the joint are used to establish a compressive force in the longitudinal direction, which increase the shear capacity. In order to simulate this behaviour using a single joint element both ends are supported, i.e equilibrium is not required for the generalised nodal forces $q_{N 1}$ and $q_{N 2}$, see Fig. 5. The detailed model [15] and the experiments are subjected to these boundary conditions at the ends, and the single joint element may overestimate the capacity slightly due to this choice of supports.

Two interface elements [15] on either side of the joint element is used to 
simulate the keyed interface. The interfaces require two material parameters, namely cohesion and friction coefficients, which are fitted to the curve of the detailed model. The magnitude of the cohesion depends on the geometry of the keys as well as the reinforcement layout.

For the analysis, an efficiency factor of $\nu=1$ has been used, i.e. no reduction in the compressive strength. This is due to the fact that the primary load carrying mechanisms is direct strut action (as found in the detailed numerical mode [15]) with very deep strut inclination. $\nu=1$ was also adopted by Nielsen and Hoang [1] and Herfelt et al. [15].

\subsection{Analysis}

The calculations have been carried out using the geometry and layout of the tests by Hansen and Olesen [6] and Fauchart and Cortini [7]. The normalised shear capacity is plotted as a function of the mechanical reinforcement ratio, which we define as:

$$
\Phi=\frac{\sum A_{s u} f_{Y u}}{t l f_{c}}
$$

where $A_{s u}$ is the cross sectional area of the U-bars, $t$ is the thickness of the joint (see Fig. 2), and $l$ is the total length of the considered joint.

Hansen and Olesen [6] investigated the behaviour of keyed joints with different reinforcement layouts. Some of the specimens featured a significant distance between the U-bars (see Fig. 9) and yielded a lower shear capacity compared to other experiments with similar reinforcement ratios. Moreover, Hansen and Olesen [6] reported that the concrete core of the specimens was completely destroyed at failure. A friction coefficient of $\mu=0.6$ is used for all specimens by Hansen and Olesen [6], while $\mu=0.75$ is used for the experiments by Fauchart and Cortini [7]. The cohesion coefficient varies between 1 and $2 \mathrm{MPa}$.

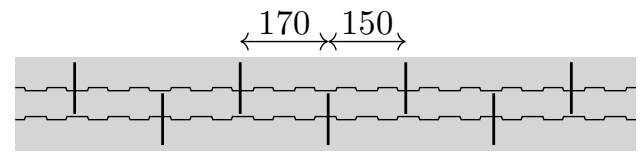

(a) Wall Joint: Specimen 24

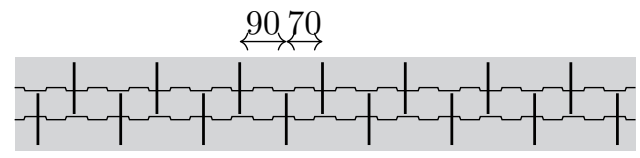

(b) Wall Joint: Specimen 26

Figure 9: The two specimens with a significant distance between the U-bars tested by Hansen and Olesen [6], measurements in millimetres. 
The specimens seen in Fig. 9 have a length of $l=1200 \mathrm{~mm}$, a width of $b=50 \mathrm{~mm}$, an overlap of $o=30 \mathrm{~mm}$, and 14 keys total. The keys have a depth of $6 \mathrm{~mm}$, a length of $h_{2}=40 \mathrm{~mm}$, and a spacing of $h_{1}=40 \mathrm{~mm}$ (see Fig. 2). As seen in Fig. 9, the two specimens featured a significant distance between the loop reinforcement, while the U-bars were placed with a mutual distance of $10 \mathrm{~mm}$ for the remaining specimens by Hansen and Olesen [6]. For specimens 24 and 26, the results are illustrated as a function of the reinforcement degree in Fig. 10, and the results for all specimens $[6,7]$ are shown in Fig. 11 and listed in Tab. 1 and 2.

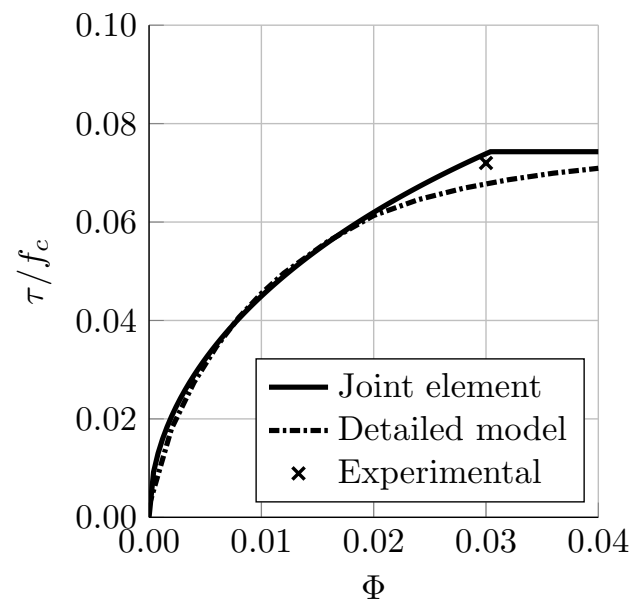

(a) Specimen 24

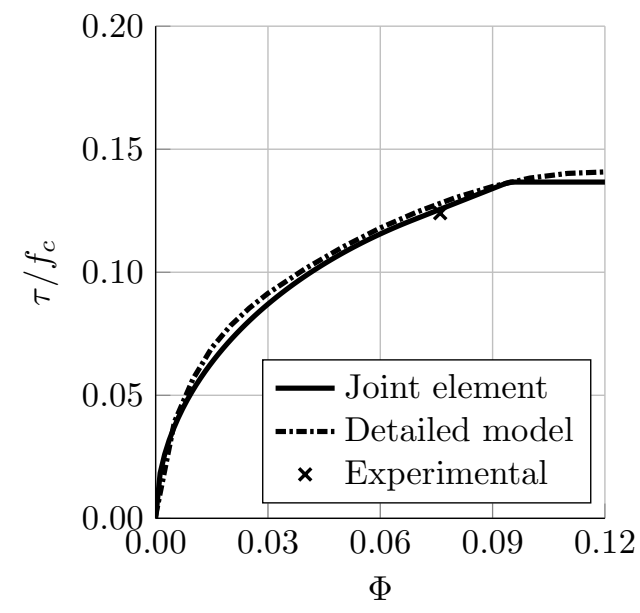

(b) Specimen 26

Figure 10: Comparison of results obtained by the joint element and the detailed model using several thousand elements. Experimental results for specimens 24 (a) and 26 (b) by $[6]$.

The cohesion is taken as $c=1.60 \mathrm{MPa}$ for specimen 24 and $c=1.90 \mathrm{MPa}$ for specimen 26. Fig. 10 shows that the joint element can capture the same behaviour as the detailed model. The plane interface elements can represent the same behaviour as keyed interfaces of the detailed model and the experiments, while the submodel yield criterion predicts an excellent estimate of the plateau, i.e. the upper limit for shear capacity. The joint element overestimates the capacity of specimen 24 slightly compared to the detailed model, which may be due to the choice of supports.

Fig. 11 shows the results of the joint element with the submodel yield criterion plotted against the results of the detailed model and the experimen- 


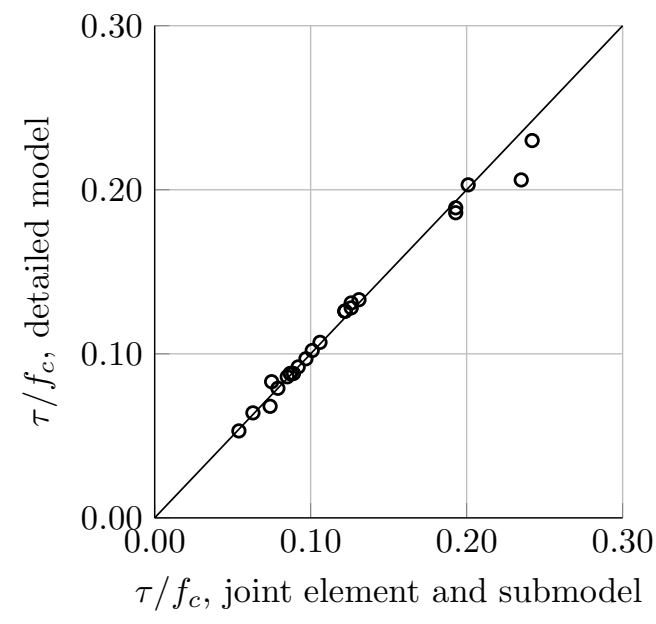

(a)

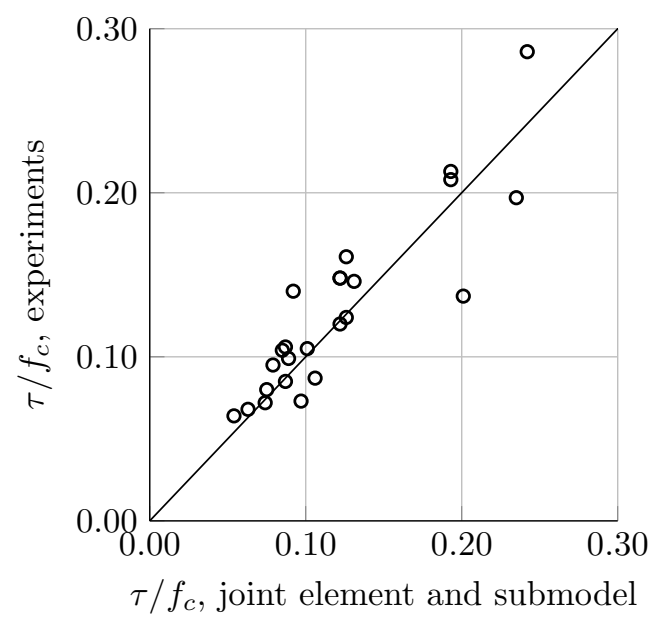

(b)

Figure 11: Analysis using the proposed joint element: a) Comparison with the detailed model [15], b) comparison with experimental results $[6,7]$.

tal results. Again, the single joint element predicts a satisfactory estimate of the load carrying capacity; only a negligible difference is observed in Fig. 11(a). The joint element predicts a slightly larger shear capacity than the detailed model for some of the experiments, which may be due to the assumptions regarding the supports. Fig. 11(b) shows that the joint element gives satisfactory results compared to experimental data.

It has been shown that the multiscale joint model captures the same behaviour as the detailed model and predicts an excellent estimate of the shear capacity. In this context, it should be mentioned that the multiscale formulation leads to a small optimisation problem which can be solved in a fraction of a second. For the detailed model, on the other hand, CPU time and problem size are several magnitudes larger as seen in Tab. 3. The computational time shown in Tab. 3 is the average of 10 runs. The performance is tested on a desktop computer with 12 GB RAM memory and an Intel Xeon CPU W3565 with 8 CPUs and $3.2 \mathrm{GHz}$ clock frequency. The optimisation is performed in MatLab using the commercial solver MOSEK [26]. Based on the required computational time, it is evident that in practice, the joint element model has to be used if entire precast concrete structures should be modelled and analysed within the limited time frame that usually is available in real design projects. 
Table 1: Data and results of experiments by Hansen and Olesen [6], the numerical model [15], and the proposed joint element.

\begin{tabular}{ccccccc}
\hline Specimen & $\begin{array}{c}f_{c} \\
{[\mathrm{MPa}]}\end{array}$ & $\begin{array}{c}\Phi \\
{[-]}\end{array}$ & $\begin{array}{c}u \\
\mathrm{~mm}\end{array}$ & $\begin{array}{c}\text { Experimental } \\
\tau / f_{c}\end{array}$ & $\begin{array}{c}\text { Detailed } \\
\tau / f_{c}\end{array}$ & $\begin{array}{c}\text { Joint element } \\
\tau / f_{c}\end{array}$ \\
\hline 01 & 29 & 0.013 & - & 0.064 & 0.053 & 0.054 \\
02 & 32 & 0.030 & - & 0.095 & 0.079 & 0.079 \\
03 & 32 & 0.055 & - & 0.105 & 0.102 & 0.101 \\
04 & 16 & 0.061 & - & 0.087 & 0.107 & 0.106 \\
05 & 53 & 0.018 & - & 0.068 & 0.064 & 0.063 \\
12 & 25 & 0.043 & - & 0.140 & 0.092 & 0.092 \\
13 & 23 & 0.095 & - & 0.146 & 0.133 & 0.131 \\
14 & 25 & 0.039 & - & 0.099 & 0.088 & 0.089 \\
18 & 27 & 0.049 & - & 0.073 & 0.097 & 0.097 \\
29 & 17 & 0.188 & - & 0.137 & 0.203 & 0.201 \\
\hline 23 & 31 & 0.025 & 10 & 0.080 & 0.083 & 0.075 \\
24 & 26 & 0.030 & 150 & 0.072 & 0.068 & 0.074 \\
25 & 24 & 0.076 & 10 & 0.161 & 0.131 & 0.126 \\
26 & 24 & 0.076 & 70 & 0.124 & 0.128 & 0.126 \\
27 & 15 & 0.139 & 10 & 0.213 & 0.189 & 0.193 \\
28 & 13 & 0.235 & 10 & 0.286 & 0.230 & 0.242 \\
\hline
\end{tabular}

Table 2: Data and results of experiments by Fauchart and Cortini [7], the numerical model [15], and the proposed joint element.

\begin{tabular}{ccccccc}
\hline Specimen & $\begin{array}{c}f_{c} \\
{[\mathrm{MPa}]}\end{array}$ & $\begin{array}{c}\Phi \\
{[-]}\end{array}$ & $\begin{array}{c}u \\
\mathrm{~mm}\end{array}$ & $\begin{array}{c}\text { Experimental } \\
\tau / f_{c}\end{array}$ & $\begin{array}{c}\text { Detailed } \\
\tau / f_{c}\end{array}$ & $\begin{array}{c}\text { Joint element } \\
\tau / f_{c}\end{array}$ \\
\hline 5 & 20 & 0.049 & 10 & 0.106 & 0.088 & 0.087 \\
6 & 20 & 0.049 & 10 & 0.085 & 0.088 & 0.087 \\
7 & 20 & 0.096 & 10 & 0.120 & 0.126 & 0.112 \\
8 & 20 & 0.246 & 10 & 0.197 & 0.206 & 0.235 \\
9 & 20 & 0.047 & 10 & 0.104 & 0.086 & 0.085 \\
10 & 20 & 0.096 & 10 & 0.148 & 0.126 & 0.122 \\
11 & 20 & 0.096 & 10 & 0.148 & 0.126 & 0.122 \\
12 & 20 & 0.191 & 10 & 0.208 & 0.186 & 0.193 \\
\hline
\end{tabular}


Table 3: Comparison of problem data for the joint element and detailed model.

\begin{tabular}{lcc}
\hline & Joint element & Detailed model $[15]$ \\
\hline Number of equilibrium elements & 3 & 20,024 \\
Number of variables & 302 & $1,364,509$ \\
Number of linear constraints & 388 & $1,305,890$ \\
Number of conic constraints & 44 & 134,472 \\
Optimisation time & $0.022 \mathrm{~s}$ & $97.89 \mathrm{~s}$ \\
\hline
\end{tabular}

\section{Conclusion}

An equilibrium joint element for modelling of keyed shear joints has been presented. The scope of the joint element is to be able to assess the strength of shear walls in precast concrete buildings, which is highly dependent on the shear capacity of in-situ cast joints. The main purpose of these joints is to transfer shear, which is done by mobilising the reinforcement, i.e. loop reinforcement and locking bar. The reinforcement layout, however, may introduce local mechanisms inside the concrete core of the joint. A multiscale approach to this problem is taken, and a equilibrium joint element as well as submodel yield criterion have been proposed.

Three modified stringer models form the basis of the submodel. The resulting stress states are obtained by adding the stress states of the three stringer models, which are checked against a suitable yield condition. The submodel is formulated for second-order cone programming, which can be solved efficiently using interior point methods.

The joint element and submodel are validated by comparison to a detailed numerical model based on finite element limit analysis. Excellent agreement between the two models is found, and the joint element captures the same behaviour as the detailed model. The joint element also predicts a satisfactory estimate of the shear capacity when compared to experimental data. The results obtained from the joint element are generally satisfactory, and the fact that the joint element only poses a small mathematical problem, which can be solved in a fraction of a second, makes it more relevant for practical applications than the detailed model presented in [15]. It can be concluded that the proposed multiscale model makes it possible to model real size precast structures while accounting for the complex behaviour of the in-situ cast joints. 


\section{Acknowledgement}

The authors would like to thank the ALECTIA Foundation and Innova-

tion Fund Denmark for financial support of the research presented in this paper.

\section{References}

[1] M. P. Nielsen, L. C. Hoang, Limit Analysis and Concrete Plasticity, Third Edition, Taylor \& Francis, 2010.

[2] A. Muttoni, J. Schwartz, B. Thürlimann, Design of Concrete Structures With Stress Fields, Springer, 1997.

[3] A. Muttoni, M. F. Ruiz, F. Niketic, Design versus assessment of concrete structures using stress fields and strut-and-tie models, ACI Structural Journal 112 (5) (2015) 605.

[4] M. F. Ruiz, A. Muttoni, On development of suitable stress fields for structural concrete, ACI Structural journal 104 (4) (2007) 495.

[5] European Committee for Standardization, En 1992-1-1 eurocode 2: Design of concrete structures - part 1-1: General rules and rules for buildings (2005).

[6] K. Hansen, S. O. Olesen, SBI-Report 97: Keyed Shear Joints, Statens Byggeforskningsinstitut, 1976.

[7] J. Fauchart, P. Cortini, Étude expérimentale de joints horizontaux entre panneaux préfabriqués pour murs de batiments, Annales de LInstitut Technique du Batiment et dês Travaux Publics 300 (1972) 86-103.

[8] S. H. Rizkalla, R. L. Serrette, J. S. Heuvel, E. K. Attiogbe, Multiple shear key connections for precast shear wall panels, Prestressed Concrete Institute, 1989.

[9] H. B. Joergensen, L. C. Hoang, Tests and limit analysis of loop connections between precast concrete elements loaded in tension, Engineering Structures 52 (2013) 558-569. 
[10] B. C. Jensen, On the ultimate load of vertical, keyed shear joints in large panel buildings, in: Symposium on Bearing Walls in Warsaw, Vol. 8, 1975, p. 13th.

[11] J. Christoffersen, Ultimate capacity of joints in precast large panel concrete buildings, Ph.D. thesis, Technical University of Denmark (1997).

[12] fib bulletin 43, Structural connections for precast concrete buildings (2008).

[13] Y. Kaneko, H. Mihashi, Analytical study on the cracking transition of concrete shear key, Materials and Structures 32 (3) (1999) 196-202.

[14] M. A. Issa, H. A. Abdalla, Structural behavior of single key joints in precast concrete segmental bridges, Journal of Bridge Engineering 12 (2007) 315-324.

[15] M. A. Herfelt, P. N. Poulsen, L. C. Hoang, J. F. Jensen, Numerical limit analysis of keyed shear joints in concrete structures, Structural Concrete 17 (3) (2016) 481-490.

[16] P. N. Poulsen, L. Damkilde, Limit state analysis of reinforced concrete plates subjected to in-plane forces, International Journal of Solids and Structures 37 (2000) 6011-6029.

[17] R. Hill, On the state of stress in a plastic-rigid body at the yield point, The London, Edinburgh, and Dublin Philosophical Magazine and Journal of Science 42 (331) (1951) 868-875.

[18] D. Drucker, W. Prager, H. Greenberg, Extended limit design theorems for continuous media, Quarterly of Applied Mathematics 9 (1952) 381389.

[19] E. Anderheggen, H. Knöpfel, Finite element limit analysis using linear programming, International Journal of Solids and Structures 8 (1972) $1413-1431$.

[20] S. W. Sloan, Lower bound limit analysis using finite elements and linear programming, International Journal for Numerical and Analytical Methods in Geomechanics 12 (1988) 61-77. 
[21] S. Krenk, L. Damkilde, O. Høyer, Limit analysis and optimal design of plates with equilibrium elements, Journal of Engineering Mechanics 120 (1994) 1237-1254.

[22] K. Krabbenhoft, L. Damkilde, Lower bound limit analysis of slabs with nonlinear yield criteria, Computers \& structures 80 (27) (2002) 20432057.

[23] A. Makrodimopoulos, C. Martin, Lower bound limit analysis of cohesivefrictional materials using second-order cone programming, International Journal for Numerical Methods in Engineering 66 (4) (2006) 604-634.

[24] C. Bisbos, P. Pardalos, Second-order cone and semidefinite representations of material failure criteria, Journal of Optimization Theory and Applications 134 (2) (2007) 275-301.

[25] K. Krabbenhøft, A. Lyamin, S. Sloan, Formulation and solution of some plasticity problems as conic programs, International Journal of Solids and Structures 44 (5) (2007) 1533-1549.

[26] MOSEK ApS, The MOSEK optimization toolbox for MATLAB manual. Version 7.1 (Revision 33) (2015).

URL https://www.mosek.com/

[27] E. D. Andersen, C. Roos, T. Terlaky, On implementing a primal-dual interior-point method for conic quadratic optimization, Mathematical Programming 95 (2) (2003) 249-277.

[28] S. P. Boyd, L. Vandenberghe, Convex Optimization, Cambridge University Press, 2004.

[29] F. Alizadeh, D. Goldfarb, Second-order cone programming, Mathematical programming 95 (1) (2003) 3-51.

[30] M. S. Lobo, L. Vandenberghe, S. Boyd, H. Lebret, Applications of second-order cone programming, Linear algebra and its applications 284 (1) (1998) 193-228.

[31] L. Damkilde, J. Olsen, P. N. Poulsen, A program for limit state analysis of plane, reinforced concrete plates by the stringer method, Bygningsstatiske Meddelelser 65 (1). 